\title{
Author Correction: Eya3 partners with PP2A to induce c-Myc stabilization and tumor progression
}

Lingdi Zhang ${ }^{1}$, Hengbo Zhou ${ }^{2,3}$, Xueni Li ${ }^{1}$, Rebecca L. Vartuli ${ }^{2,4}$, Michael Rowse ${ }^{5}$, Yongna Xing ${ }^{5}$, Pratyaydipta Rudra6, Debashis Ghosh ${ }^{6}$, Rui Zhao ${ }^{1,4}$ \& Heide L. Ford ${ }^{1,2,3,4}$

Correction to: Nature Communications, https://doi.org/10.1038/s41467-018-03327-4, published online 13 March 2018

In the original version of this Article, the title of the legend to Fig. 7 incorrectly read 'Knockdown of B55a increases breast cancer metastasis' instead of 'Knockdown of B55a decreases breast cancer metastasis'. This has now been corrected in both the PDF and HTML versions of the Article.

Published online: 17 September 2018 reproduction in any medium or format, as long as you give appropriate credit to the original author(s) and the source, provide a link to the Creative Commons license,
and indicate if changes were made. The images or other third party material in this article are included in the article's Creative Commons license, unless indicated otherwise in a credit line to the material. If material is not included in the article's Creative Commons license and your intended use is not permitted by statutory regulation or exceeds the permitted use, you will need to obtain permission directly from the copyright holder. To view a copy of this license, visit http://creativecommons.org/licenses/by/4.0/.

(C) The Author(s) 2018

\footnotetext{
${ }^{1}$ Department of Biochemistry and Molecular Genetics, University of Colorado Denver Anschutz Medical Campus, Aurora 80045, CO, USA. ${ }^{2}$ Department of Pharmacology, University of Colorado Anschutz Medical Campus, Aurora 80045, CO, USA. ${ }^{3}$ Cancer Biology Program, University of Colorado Anschutz Medical Campus, Aurora 80045, CO, USA. ${ }^{4}$ Molecular Biology Program, University of Colorado Anschutz Medical Campus, Aurora 80045, CO, USA. ${ }^{5}$ McArdle Laboratory for Cancer Research, School of Medicine and Public Health, University of Wisconsin-Madison, Madison 53705, WI, USA. ${ }^{6}$ Department of Biostatistics and Informatics, University of Colorado Anschutz Medical Campus, Aurora 80045, CO, USA. These authors contributed equally: Lingdi Zhang, Hengbo Zhou. Correspondence and requests for materials should be addressed to R.Z. (email: rui.zhao@ucdenver.edu) or to H.L.F. (email: heide.ford@ucdenver.edu)
} 